\title{
Research Progress of Traditional Chinese Medicine Constitution Theory in Patients with Cervical Spondylosis
}

\author{
Hui-Xin DING, Chun-Guang YU*, Ling WANG, Chun-Hua ZHANG, Pei-Yao LI \\ School of Nursing, Beijing University of Chinese Medicine, Beijing 100029, China
}

\begin{abstract}
This paper expounds the theory of traditional Chinese medicine (TCM) constitution applied to patients with cervical spondylosis, in order to clarify the distribution characteristics of TCM constitution of patients with cervical spondylosis. And to analyze the correlation between TCM constitution theory and cervical spondylosis is helpful to provide ideas for integrated nursing care of TCM and western medicine in patients with cervical spondylosis, and provide the basis for the construction of health management program and the evaluation of health promotion effect.
\end{abstract}

Key Words: Cervical spondylosis; Theory of TCM constitution; Summary; Integrative nursing

\section{Introduction}

With the continuous improvement of TCM constitution theory, studies on the application of TCM constitution theory to cervical spondylosis provide a new way to treat cervical spondylosis by individual treatment. Cervical spondylosis belongs to the category of "arthralgiasyndrome", which is a frequently occurring clinical disease. The case rate of it is more than $64.52 \%$, and the incidence presents a younger trend ${ }^{[1]}$. The main pathogenic factors include deficiency of kidney-qi, fatigue, internal injury, six exogenous pathogenic factors, accidental injury and so on. The pathogenesis of cervical spondylosis is the degenerative lesion of intervertebral disc caused by deficiency of kidney-qi, in addition, further lesions such as secondary bone injury, muscle injury and joint enlargement may cause pain in the neck and shoulder joint, vertigo caused by vertebral artery compression, and

\section{"Corresponding Author:}

Chun-Guang YU, School of Nursing, Beijing University of Chinese Medicine, Beijing 100029, China E-mail: bjchunguang@163.com

Received:April 19 2019, Revised: May 10 2019,Accepted: May 30 2019, Online: August 152019 flaccid syndrome caused by neuron injury ${ }^{[2]}$. According to the theory of TCM constitution, the thought of "preventive treatment of disease" can be infiltrated into the prevention and cure strategy of susceptible constitution type, which can provide the basis for the evaluation of health management effect of patients with cervical spondylosis in the next step. In this paper, we searched the Chinese biomedical literature database (CBM), China National Knowledge Infrastructure (CNKI), WanFang data knowledge service platform and VIP database as the sources of data retrieval, summarized as follows.

\section{Application of Theory of TCM Constitution in Cervical Spondylosis}

The constitution is a synthetic and relatively stable inherent characteristic that is integrated in terms of morphological structure, physiological function, and mental state in the process of human life, which is 
formed on the basis of innate endowment and acquired by practice. It is the human personality characteristic which adapts to the natural and social environment during the development and growth of human beings. Constitution shows individual differences in structure, function, metabolism, and response to external stimuli, susceptibility to certain causes and diseases, and a certain tendency in the course of disease progression ${ }^{[3]}$. The discussion of TCM constitution began with "The Canon of Internal Medicine", after inheriting the thoughts of ancient doctors modern doctors put forward many methods of classification of the constitution in combination with clinical practice. At present, there are two main methods to classify the constitution of patients with cervical spondylosis. One is Nine categories of TCM constitution, the other is "medicine person" constitution.

\section{Nine categories of TCM constitution}

In the process of constructing the theory system of TCM constitution, professor Wang Qi put forward "the diagnosis and treatment mode of differentiation of constitution, differentiation of disease and differentiation of syndrome", through long-term clinical practice and scientific experimental work of TCM. Professor Wang Qi emphasized that "constitution is the background of disease and syndrome". Constitution differentiation and treatment means that the effects of constitutional differences on the emergence, development, prognosis and treatment plan of the disease should be fully taken into account in the course of treatment of the disease. In other words, Patients with the different constitution should be treated individually in terms of prescription, choice of drugs and dosage ${ }^{[4]}$. After 30 years, Professor Wang Qi has established nine categories of TCM constitution classification by means of literature research, epidemiological investigation and analysis, combined with clinical observation. Professor Wang Qi puts forward "theory on divisibility of constitution", "theory on relationship between constitution and disease" and "theory on adjustable constitution", which has been recognized by the Chinese Academy of Traditional Chinese Medicine as the national accepted standard "classification and judgment of TCM constitution" ${ }^{,[5]}$. Nine kinds of constitution include yinyang harmony constitution, qi-deficiency constitution, yang-deficiency constitution, yin-deficiency constitution, phlegm-dampness constitution, damp-heat constitution, blood-stasis constitution, qi-stagnation constitution, and specific endowment constitution.

\section{“Medicine person" constitution}

Professor Huang attaches importance to the differentiation of symptoms and signs of the constitution, explores the relationship between constitution and medication, finds out that there is an obvious connection between constitution and medication, who inherited Zhang Zhongjing's thought of physique theory, and drawn lessons from Japan's constitution thoughts. Professor Huang puts forward the constitution concept of "medicine person" and "prescription person" combined with his own experience, which is named by longterm use of TCM drug or prescription ${ }^{[6]}$. "Medicine person" includes "ramulus cinnamomi constitution", "ephedra constitution", "bupleurum constitution", "rhubarb constitution", "pinellia constitution", "ginseng constitution", "angelica constitution", "peony constitution" and so on. "Prescription person" includes "warming meridiandecoction constitution", "Sanhuangxiexin decoction constitution", "liquorice decoction constitution", "cassia twig and keel oyster decoction constitution"and so on ${ }^{[7-8]}$.

\section{Features of Constitution Distribution in Patients with Cervical Spondylosis}

\section{Focus on biased physical fitness}

The TCM constitution of patients with cervical spondylosis is a mainly comprehensive constitution. Zhao et al. ${ }^{[9]}$ investigated the TCM constitution of inpatients with cervical spondylosis by using the questionnaire of TCM constitution scale and general data questionnaire, whose results showed that the TCM constitution of cervical spondylosis patients had comprehensive constitution aggregation phenomenon. Bao et al. ${ }^{[10]}$ explored the distribution characteristics of TCMconstitution types in patients with cervical spondylosis through a controlled trial, in which taking 200 patients diagnosed as cervical spondylosis in the physical 
examination center as the research object compared with 200 healthy people randomly selected as the control group. The constitution of the patients was measured by TCM constitution classification and judgment scale.The results showed that $72.5 \%$ of cervical spondylosis patients had a comprehensive constitution. Chen et al. ${ }^{[11]}$ selected 200 perimenopause women diagnosed as cervical spondylosis in the physical examination center as the case group and 96 perimenopause healthy women as the control group. The results showed that the proportion of yinyang harmony constitution was lower than that of healthy women, while the proportion of the comprehensive constitution was higher.

\section{Existence of comprehensive constitution}

The TCM comprehensive constitution refers to one main kind of constitution accompanying other types of constitution. The diversity of physique formation factors leads to the difference in physiology and pathology. On the one hand, the various physical factors that form constitution change with the progress of life, on the other hand, the effects and influences of various pathological factors, which lead to the constitution type changing or appearing comprehensive constitution ${ }^{[12]}$. Among the 200 subjects included in the study by Bao et $a l .^{[13]}, 27.5 \%$ are of yin-yang harmony constitution, $72.5 \%$ of comprehensive constitution, $45.5 \%$ of simple comprehensive constitution, and $27 \%$ of both of them have two types of comprehensive constitution, $5.5 \%$ of them have three types of comprehensive constitution, and four kinds of comprehensive constitution accounted for $5.5 \%$. $\mathrm{Su}^{[14]}$ took 90 cases of cervical spondylosis due to yang-deficiency and qi-deficiency as the objects of study, and the first three types of comprehensive constitution were yang-deficiency accompanied with the blood-stasis constitution, yang-deficiency accompanied with the qistagnation constitution, yang deficiency accompanied with qi-deficiency and qi-stagnation constitution.

\section{Distribution of major constitution}

The constitution distribution of patients with cervical spondylosis is characterized by yang-deficiency constitution. Chen et al. ${ }^{[11]}$ studied on women with cervical spondylosis during perimenopause period as the study object, the distribution of TCM constitution of yangdeficiency constitution is the most. Zhao et al. ${ }^{[9]}$ found that the proportion of yang-deficiency constitution, bloodstasis constitution and phlegm-dampness constitution in patients with cervical spondylosis was higher, and there was statistical significance in comparison with the TCM constitution among the general population. Besides, the yang-deficiency constitution, the blood-stasis constitution, and the phlegm-dampness constitution have statistical significance in gender difference. Due to the differences in morphology, physiology, material metabolism, and heredity between men and women, different constitution characteristics of men and women have been formed. Fan et $a l .^{[15]}$ selected 90 patients with cervical spondylosis, compared with 90 healthy check-ups in the same hospital, and explore the proportion of constitution distribution between patients with cervical spondylosis and healthy people. The same results as the former were that yangdeficiency constitution, blood-stasis constitution, and phlegm-dampness constitution had a higher proportion of abnormal constitution distribution. But except yangdeficiency constitution as the main, there are differences in secondary constitution. $\mathrm{Su}^{[14]}$ found that apart from yangdeficiency constitution, the frequency of qi-stagnation constitution, blood-stasis constitution, and qi-deficiency constitution appeared in the first three places.Pan et al. ${ }^{[16]}$ used Chinese medicine meridian detector to identify the constitution of 80 patients with cervical spondylosis in order to study the constitution types of cervical spondylosis. The results showed that of 80 patients with cervical spondylosis, the incidence of yang-deficiency constitution was the highest, and accounted for $23.75 \%$. Besides, the ratio of blood-stasis constitution, qi-deficiency constitution, and the damp-heat constitution was also high.

In addition, at present the application of TCM constitution theory to cervical spondylosis is influenced by different research objects and methods, which leads to the different findings of the main constitution distribution of cervical spondylosis patients. It is believed that the characteristics of the constitution distribution of cervical spondylosis patients are mainly qi-deficiency constitution. Zhong et al ${ }^{[17]}$ applied the questionnaire of cervical spondylosis and the questionnaire of TCM constitution 
scale to conduct a cross-sectional field survey and made stratified cluster sampling according to the nature of the administrative organs, such as banks, schools, hospitals, and other units. Zhong selected workers from 18 to 50 years of age who were engaged in low-head ambush cases when working as the research object, Zhong actually investigated 1739 cases of subjects, including 286 cases of cervical spondylosis. It was found that the proportion of yin-yang harmony constitution in non-cervical spondylosis population was higher than that in cervical spondylosis population, and in the abnormal constitution, the proportion of qi-deficiency constitution and damp-heat constitution in cervical spondylosis population was higher than that in the non-cervical spondylotic group.

There are similarities and differences among different constitution classification methods. Yao et al. ${ }^{[18]}$ analyzed the relationship between Professor Wang Qi's nine types of TCM constitution and that of Professor Huang's "prescription person" and "medicine person". It was found that qi-deficiency constitution comparing with astragalus mongholicus constitution and Guizhi constitution, phlegm-dampness constitution comparing with ephedra constitution, qi-stagnation constitution and blood-stasis constitution comparing with radix bupleuri constitution, damp-heat constitution comparing with rheum officinale constitution in the common symptoms, signs and the application of prescription and medicine. The difference is that the yin-yang harmony constitution and specific endowment constitution in the nine types of the constitution of TCM need to be treated individually by the differentiation of syndrome. Xu et al. ${ }^{[19]}$ applied Professor Huang's constitution-based syndrome differentiation theory ${ }^{[20-22]}$ in cervical spondylosis. The results showed that there were 19 cases of radix bupleuri constitution, 28 cases of astragalus mongholicus constitution, 18 cases of ephedra constitution, 16 cases of cassia twig constitution, 13 cases of pinellia ternata constitution, 5 cases of rhubarb constitution, 5 cases of yang-heat constitution, 10 cases of kudzu vine constitution, and 1 case of ginseng constitution. It can be seen that $\mathrm{Xu}$ et al's study results are consistent with Zhong et al.'s research results, in which cervical spondylosis is dominated by the qi-deficiency constitution, followed by the phlegm-dampness constitution.

\section{Relationship Between TCM Constitution and Patients with Cervical Spondylosis}

\section{TCM constitution is associated with the attack of cervical spondylosis}

There was a positive correlation between cervical spondylosis and yang-deficiency constitution, Related studies show that the risk of cervical spondylosis was 1.329 times higher than that of yin-yang harmony constitution, and the risk was significantly higher ${ }^{[10,13]}$. This is the same as the results of Chen ${ }^{[11]}$, who found that yangdeficiency constitution is positively associated with the cervical spondylosis women in the perimenopausal period. The risk of cervical spondylosis in the perimenopausal women with yang-deficiency constitution is significantly higher than that of the yin-yang harmony constitution, and the OR value is 1.329 . This is in line with the viewpoint put forward by $\mathrm{Hu}$ et $a .^{[23]}$, who thinks that"different types of constitution determine the tendency of different individuals to certain causes, special susceptibility to disease and the tendency of pathological processes". Yangdeficiency constitution is mainly due to deficiency of yang-qi, especially deficiency of spleen-yang and kidneyyang, which is a pathological state of viscera dysfunction or weakness, metabolic activity decline, and heat deficiency ${ }^{[24]}$. Cervical spondylosis, as a chronic disease caused by multiple factors, can also affect the constitution types of patients with cervical spondylosis. The ancient book of "Ji Sheng Prescription--Arthralgia Syndrome" points out that all disease cause of arthralgia-syndrome is body deficiency, striae are empty and sparse, easily affected by wind, cold and dampness". Natural deficiency, forced work, and other reasons lead to the loss of vital-qi and the decline of viscera function, thereby affecting the distribution, transportation and metabolism of body fluid, further resulting in water and wetness stagnated within body, accumulation of phlegm and dampness, phlegmdampness blocking qi and blood circulation, eventually make bones, muscles lost in nourishment. By Logistic regression analysis, Zhong ${ }^{[25]}$ found that the risk factors of cervical spondylosis include long lying time, improper working posture, age, the discomfort of sleeping pillow, improper life posture, not taking part in physical exercise 
and so on. This is close to the results of Zhu et al.'s crosssectional field investigation ${ }^{[26]}$, an investigation which discussed the main influencing factors of yang-deficiency constitution by single factor and multiple regression analysis. It was concluded that the most influential factor yang deficiency constitution was a chronic disease, followed by the female, lack of movement, hot diet, underweight, light diet, non-breastfeeding after birth ${ }^{[26-28]}$.

The incidence of cervical spondylosis is related to constitution type and meridian characteristics. Pan et al. ${ }^{[16]}$ used Chinese medicine meridian detector to identify constitution and detect meridian in 80 patients with cervical spondylosis in order to study on constitution types and meridian characteristics of cervical spondylosis. The results showed that the abnormal rate of the small intestine meridian was $13.71 \%$, but there was no obvious deficiency and excess bias. In the other meridians with higher abnormal rate, the energy of the Sanjiao meridian, liver meridian and large intestine meridian was on the low side, but only the energy value of the bladder meridian was higher. It is recorded in the TCM ancient books "Plain Questions (Suwen)" that the liver is in charge of the tendons, which means that the deficiency of liver-blood makes the fascia of the human body not fully nourished, and then leads to the tension of the muscles, finally leads to the neck and shoulder muscle pain, stiffness, limited movement and so on. What's more, it is recorded in the TCM ancient book "Canon of Internal Medicine" that "liver governing conveyance and dispersion". The function of the liver can convey and disperse the normal body, which can regulate the movement of qi and promote fluid metabolism and blood movement. If the liver function is abnormal, it will cause the cervical meridian qi-blood disorder, and eventually lead to the occurrence of cervical spondylosis ${ }^{[29]}$. Therefore, the abnormal proportion of the above 5 meridians is higher than other meridians. From the analysis of the deficiency and excessive syndrome of the single meridian, it can be seen that the Sanjiao meridian and the large intestine meridian follow the upper arm, if the above two meridians are manifested as "deficiency syndrome", they may cause clinical symptoms of numbness and weakness at the upper limbs passing through the line, which is in line with the clinical symptoms of cervical spondylosis. On the other hand, if the liver is manifested as "deficiency syndrome", then the function of "liver governing tendon" and "liver governing conveyance and dispersion" will be abnormal, which further leads to insufficiency of liver-blood and liver essence and disorder of qi-blood, and eventually leads to neck discomfort. So the values of blood resistance of the above four meridians are all on the low side, and "deficiency syndrome" as the main manifestation. It is recorded in the TCM ancient book "Treatise on Febrile Diseases--Taiyang Disease" that "clinical manifestation of Taiyang Disease is characterized by floating pulse, stiff pain in the head and neck and fear of cold". On the one hand, The bladder meridian of foot-taiyang follows the back of the neck, three evils of wind, cold and dampness first invade the meridian of the taiyang when the body is weak, and then cause neck discomfort, on the other hand, the invasion of excessive evilleads to cervical discomfort. Therefore, the electrical value of bladder meridian acupuncture points are on the high side, and the main manifestation is "excessive syndrome" $"$ "16]

The occurrence and development of cervical spondylosis are related to the TCM constitution and seven emotions, so we should pay attention to the patient's constitution adjustment and emotional nursing in the course of prevention and nursing of cervical spondylosis. Zhao et al. ${ }^{[30]}$ adopted TCM constitution scale and sevenemotion background questionnaire to investigate 106 inpatients with cervical spondylosis. The results showed that patients with cervical spondylosis had anger, joy, worry as the dominant emotion and less panic. Besides, the male was dominated by anger and joy. Women are dominated by anger and worry. With more than 30 years of in-depth researches, Wang Qi has summed up four basic principles, which is "the theory of constitution process", "the theory of the constitution of mind and body", "the theory of environmental restraint", and "the theory of natural endowment inheritance". The main idea is that constitution is a combination of specific physical quality and certain psychological quality, and is the concrete expression of the thought of "the unity of the body and the spirit" in the theory of TCM constitution. Wang ${ }^{[31]}$ found that different TCM constitution has different 
psychological characteristics. Qi-deficiency constitution is characterized by introversion, emotional instability, and timidity. Personality characteristic of yang-deficiency constitution is more quiet, and introverted. Phlegmdampness constitution is characterized by warm and stable, courteous, open-minded, more patient. The dampheat constitution is characterized by the more irritable. Blood-stasis constitution tends to annoyance, impatience, and forgetfulness. The character of the qi-stagnation constitution is introverted, instable, melancholy fragile, sensitive, suspicious, and susceptible to mental stimulation. An abnormal psychological state is related to the structure and physiological function of the abnormal constitution. If it can not be adjusted in time, long-term accumulation will lead to the occurrence of psychological diseases. It is recorded in the TCM ancient book "Plain Questions" that "the liver is harmfully affected by over-anger", "the heart is harmfully affected by over-joy", and "the lung is harmfully affected by over-grief". Invasion of the heart, liver, and lung can cause blood stasis, stagnation of liver-qi and obstruction of qi, which may be the pathological basis for the formation of the corresponding constitution ${ }^{[32]}$. The distribution of constitution of cervical spondylosis is yangdeficiency constitution, qi-deficiency constitution, phlegmdampness constitution, blood-stasis constitution, and qistagnation constitution. The psychological characteristics of cervical spondylosis are in line with Zhao's research results that anger, happiness, and worry are the dominant emotions in cervical spondylosis patients.

\section{Nursing of the TCM constitution can improve the clinical symptoms of cervical spondylosis}

Applying the theory of TCM constitution in patients with cervical spondylosis can effectively improve clinical symptoms of cervical spondylosis based on different constitution characteristics combined with TCM characteristic technology. TCM holds that moxibustion has the functions of warming meridian to dissipate cold, helping yang to avoid breaking down, removing blood stasis and dispersing knot. As recorded in the TCM ancient book "Elementary Medicine Acupuncture and Moxibustion", "If the medicine is not good and the curative effect of acupuncture cannot be achieved, the moxibustion method will certainly be effective". Ginger moxibustion at Dazhui point and Feishu points has always been used as an important means to treat yang-deficiency diseases such as fear of cold, cold hands and feet, and it also has the effect of obviously improving the deficiency disease. $\mathrm{Su}^{[14]}$ used a random number table method to divide 90 patients into 3 groups: observation group, non-acupoint control group, and basic treatment group. The cervical spondylosis patients with yang-deficiency constitution were treated with ginger moxibustion at Dazhui and Feishu points combined with acupuncture. The results showed that the curative effect of ginger moxibustion combined with acupuncture on yangdeficiency constitution was better than that of acupuncture alone. The Japanese Orthopaedic Association Scale (JOA)'s scores of ginger moxibustion combined with acupuncture were better than that of single acupuncture before and after treatment. There was a significant difference in Visual Analogue Scale (VAS)'s score and Orthopaedic Association Scale (JOA)'s score among the 3 groups before and after treatment. Three months after the treatment, the difference of yang-deficiency constitution's score between the observation group and the nonacupoint control group and the basic treatment group was statistically significant. It can be seen that the treatment of cervical spondylosis based on TCM constitution theory can not only relieve the symptoms of cervical spondylosis patients but also regulate the constitution, which can improve the abnormal constitution.

Nursing under the guidance of TCM constitution theory and according to different constitution characteristics of cervical spondylosis has a better curative effect in regulating constitution, relieving neck fatigue and eliminating main symptoms. $\mathrm{Xu}$ et al.$^{[22]}$ found that the treatment of cervical spondylosis by acupuncture and moxibustion at local acupoints combined with Huang's acupoints treatment based on syndrome differentiationis the better clinical effect. After 3 courses of treatment, 94 cases were cured, 30 cases were markedly effective and 6 cases were improved in 103 cases of cervical spondylosis. TCM nursing for cervical spondylosis according to the characteristics of blood-stasis constitution has a better effect. Yan et al ${ }^{[33]}$ selected 64 patients who met the criteria of cervical sub-health diagnosis and all of 
them were blood-stasis constitution according to TCM Constitution Classification and Judgment Scale and Cervical Sub-health Assessment scale. The patients were randomly divided into 2 groups: acupuncture group $(n=32)$ and routine acupuncture group $(n=32)$. According to Professor Fu Jiaying's thought of regulating blood-stasis constitution, the treatment group first selected Dazhui acupoint to prick collaterals and draw blood cupping, then selected Geshu, GanShu, Taichong, Xuehai, Sanyinjiao to carry on acupuncture. At last, acupuncture was carried out into Fengchi, Tianzhu, Jilao, Dazhu, and the method of mild reinforcing and mild reducing was carried out after Qi was obtained.And the routine treatment of cervical spondylosis selected Fengchi, Tianzhu, Bailao, Dazhu, Gansu, Sanyinjiao, Taichong points, acupuncture after developing needle sensation were all treated with the method of mild reinforcing and mild reducing, assisted by cupping in point of Qimen and Riyue. It was found that both groups were effective in the treatment of cervical sub-health, but the acupuncture and moxibustion group was better than the conventional acupuncture group in regulating blood-stasis constitution, improving neck fatigue symptoms and eliminating main symptoms.

\section{Summary}

To sum up, further researches of TCM constitution theory applied to cervical spondylosis patients need to be carried out. The present research shows that according to the characteristics of constitution distribution of cervical spondylosis patients and the correlation between TCM constitution and cervical spondylosis, individualized implementation of TCM characteristic nursing technology can effectively improve the related symptoms of cervical spondylosis. Based on the academic theory of "preventive treatment of disease", we can explore the nursing measures of the main abnormal constitution types of cervical spondylosis individually, and provide the basis for the construction of health management program and the evaluation of health promotion effect.

\section{Declaration}

The authors of this article declare they have no conflict of interest.

\section{References}

1 Wang B, Duan YP, Zhang YC, et al. Epidemiological study on the characteristics of cervical spondylosis. $\mathrm{J}$ Central South Univ (Medical Edition), 2004, 29(4): 472-474.

2 Wang L. Study on the pathogenesis of cervical spondylosis by TCM theory and literature arrangement. Fuzhou: Fujian University of Traditional Chinese Medicine, 2013.

3 Wang Q. TCM physique. Beijing: People's Health Publishing House, 2005:2.

4 Jiang M. Professor Wang Qi's syndrome differentiation-disease differentiation-syndrome differentiation combined with academic thought and clinical experience and clinical study on the treatment of chronic insomnia. Beijing: Beijing University of Traditional Chinese Medicine, 2011.

5 China Association of Chinese Medicine. TCM constitution classification and judgement (ZYYXH/ T157-2009). World J Integr Chin Western Med, 2009, 4(4): 303-304.

6 Liu XQ. A brief introduction to Huang's Constitution Theory. Liaoning J Trad Chin Med, 2008, 35(8): 1166-1167.

7 Lin Y. A Clinical study on the physique Classification elements of Huang. Guangzhou: Guangzhou Univ Trad Chin Med, 2011

8 Huang H. Ten Categories of TCM Constitution. Jiangsu: Jiangsu Science and Technology Publishing House, 1995: 3.

9 Zhao HY, Wang XM, Liu DM. Investigation on TCM constitution of patients with cervical spondylosis. J Gansu Coll Trad Chin Med, 2013, 30(5): 82-84.

10 Bao JM, Lin XJ, Chen WH, et al. Study on the distribution and correlation of TCM constitution types in patients with cervical spondylosis. Chin Med Innov, 2016, 13(13): 90:93.

11 Chen LM, Lin XJ, Hu HY, et al. Study on constitution characteristics of women with cervical spondylosis in perimenopause. Chin Med Innov, 2015, 12(14): 98101.

12 Zhu YB. Classification and evaluation of TCM 
constitution. Chin J Trad Chin Med, 2012, 27(1): 4042.

13 Bao JM, Lin XJ, Hao YT, et al. Physique characteristics and correlation analysis of patients with cervical spondylosis. Henan trad Chin Med, 2014, 34(B06): 20-21.

14 Su QG. Clinical effect of moxibustion at Dazhui and Shuangfeishu points with acupuncture on cervical spondylosis due to yang deficiency. Fuzhou: Fujian University Traditional Chinese Medicine, 2013.

15 Fan M, Li JL, Chen CL. Investigation on characteristics of TCM physique in patients with cervical spondylosis. Shenzhen J Integr Trad Chin Western Med, 2016, 26(13): 57-59.

16 Pan C, Zhang HD, Yan YX, et al. Analysis of physique identification and meridian characteristics in 80 patients with cervical spondylosis. Introd Trad Chin Med, 2016, 22(23): 36-38.

17 Zhong WH, Feng JP, Chen SJ, et al. Study on the classification of TCM constitution of cervical spondylosis among young and middle-aged workers in Fuzhou. Bone Trad Chin Med, 2011, 23(3): 30-31.

18 Yao JQ, Li YF. Relationship between 9 basic types of traditional Chinese medicine constitution and the constitution of the prescription syndrome. Trad Chin Med Forum, 2015, 30(5): 58-60.

$19 \mathrm{Xu} \mathrm{JP}, \mathrm{Li}$ YH, Li SF. Treatment of 130 cases of cervical spondylosis by syndrome differentiation of acupuncture and moxibustion constitution. Chin Folk Med, 2015, 24(13): 153.

20 Huang H. The Charm of the Recipe. Beijing: People's Health Press, 2011: 76-82.

21 Huang H. Ten Categories of TCM Constitution. Nanjing: Jiangsu Science and Technology Press, 2010.

22 Li XR, Xue BY, Mei LF. Huang's Medical Records. Beijing: People's Military Medical Press, 2013: 1718.

$23 \mathrm{Hu}$ YN, Shang XL, Yang J. The recent 5-year research progress of correlation between physique theory and disease in TCM. J Changchun Univ Trad Chin Med, 2010, 26(3): 455-457.
24 Zheng XR, Xin B, Li HY, et al. Yang deficiency physique of TCM syndrome differentiation of nourishment. J Heilongjiang Trad Chin Med, 2015, 43(3): 22-23.

25 Zhong WH. Investigation on risk factors of cervical spondylosis and physique analysis of traditional Chinese medicine among young and middle-aged workers in Fuzhou. The Proceedings of the Eleventh National Conference on Cervical Spondylosis of China Rehabilitation Medical Association. Professional Committee on Cervical Spondylosis of Chinese Rehabilitation Medical Association, 2009.

26 Zhu YB, Wang Q, Yao SL. Study on related factors of Yang deficiency quality in traditional Chinese Medicine. J Trad Chin Med, 2007, 48(12): 1113-1115.

27 Yu MY, Huang LP, Ling JH, etc. Analysis on the causes of Yang deficiency physique in Guangxi. J Pract Intern Med, 2012, 26(1): 8-9.

$28 \mathrm{Li}$ J, Wu CY, Ma ZM, et al. Study on the formation mechanism of the physique of yang deficiency in different sexes. Liaoning J Trad Chin Med, 2010, 37(1): 77-78.

29 Liu HB, Xiao YH. Discussion on the treatment of cervical spondylosis from liver theory.Chin J Basic Med Trad Chin Med, 2014, 20(5): 580-581.

30 Zhao HY, Wang XM, Liu DM. Study on the relationship between cervical spondylosis and physique of traditional Chinese medicine and the background of seven emotions. Nurs Res, 2013, (26): 2843-2845.

31 Wang Q. Classification of 9 kinds of physique types of basic TCM and their diagnostic basis. J Beijing Univ Trad Chin Med, 2005, 28(4):1-8.

32 Tang F. A study on personality and psychological characteristics of physique types in TCM. Beijing: Beijing Univ Trad Chin Med, 2010.

33 Yan LD. Clinical study of acupuncture and moxibustion in regulating and treating the sub-health of blood-stasis cervical vertebra in middle-aged and young people. Guangzhou: Guangzhou Univ Trad Chin Med, 2015. 